\title{
REPRESENTATIONS OF LOCALLY FINITE GROUPS
}

\author{
BY DAVID J. WINTER ${ }^{1}$
}

Communicated by G. B. Seligman, August 15, 1967

The purpose of this paper is to give a brief general account of the completely reducible finite-dimensional representations of a locally finite group $G$ over a given algebraically closed field $K$. Theorem 1 shows that all such representations of $G$ can be brought down to the algebraic closure $F$ in $K$ of the prime field of $K$. This reduces all further considerations in this account to countable groups. Theorem 2 characterizes the existence of a faithful completely reducible representation of $G$ of degree $n$ over $K$ in terms of the existence of such representations for appropriate finite subgroups of $G$.

Throughout the paper, $G$ denotes a locally finite group, $K$ denotes an arbitrary algebraically closed field and $F$ denotes the algebraic closure in $K$ of the prime field of $K . V$ denotes an $n$-dimensional vector space over $K$. An $F$-form of $V$ is an $F$-subspace $W$ of $V$ such that $W$ and $K$ are linearly disjoint over $K$ and $V$ is the $K$-span of $W$. (Equivalently, an $F$-form of $V$ is the $F$-span of a basis of $V$.) If $A$ is an $F$ algebra, $A_{K}$ denotes the algebra $A \otimes_{F} K$.

THEOREM 1. Let $\rho$ be a completely reducible representation of $G$ in $V$. Then $V$ has an $F$-form $W$ which is stable under the $\rho$-action of $G$ in $V$.

Proof. It suffices by complete reducibility to consider the case in which $G$ acts irreducibly in $V$.

If $G$ is finite, the assertion follows (upon passing to the group algebra of $G$ over $F$ ) from the fact that if $A$ is a finite-dimensional associative algebra over $F$, then an irreducible (finite-dimensional) $A_{K^{-}}$ module has an $F$-form stable under $A$. Since the kernel of an irreducible representation of such an $A$ contains the radical of $A$, it suffices to prove this in the case that $A$ is semisimple. But for $A$ semisimple, the assertion is obvious since:

(1) $A=\sum_{1}^{m} \oplus A_{i}$ where $A_{1}, \cdots, A_{m}$ are minimal right ideals of $A$;

(2) $A_{K}=\sum_{1}^{m} \oplus\left(A_{i}\right)_{K}$ and the $\left(A_{i}\right)_{K}$ are minimal right ideals of $A_{K}$;

(3) any irreducible $A_{K}$-module is isomorphic to one of the $\left(A_{i}\right)_{K}$ [1, Chapter IV].

Next assume that $G$ is locally finite and that $(\rho, V)$ is an irreducible representation of $G$ over $K$ of degree $n$. Then some finite subgroup $H$ of $G$ acts irreducibly in $V$. (For example let $S$ be a finite subset of $G$

\footnotetext{
1 This research was partially supported by research grant NSF-GP-4017.
} 
such that $\rho(S)$ is a maximal $K$-independent subset of $\rho(G)$, and let $H$ be the subgroup generated by $S$.) Since $H$ is finite and acts irreducibly in $V, V$ has an $F$-form $W$ stable under the action of $H$. We claim that $W$ is stable under the action of $G$. Thus let $g$ be any element of $G$ and let $I$ be the subgroup of $G$ generated by $H$ and $g . I$ is finite and acts irreducibly in $V$; and consequently $I$ also stabilizes some $F$-form, say $X$, of $V$. The $F$-algebras $A_{H}, A_{I}$ generated by $\rho(H), \rho(I)$ respectively stabilize $W, X$ respectively. By Burnside's Theorem $[1$, p. 182], $A_{H} \mid W=\operatorname{Hom}_{F}(W, W)$ and $A_{I} \mid X=\operatorname{Hom}_{F}(X, X)$. Thus $\operatorname{dim}_{F} A_{H}$ $=\operatorname{dim}_{F} A_{I}=n^{2}$. Since $A_{H} \subseteq A_{I}$, we have $A_{H}=A_{I}$. Thus $I$ stabilizes $W$. Thus $g$ stabilizes $W$. Since $g$ was chosen arbitrarily, $G$ stabilizes $W$.

Lemma. ${ }^{2}$ Let $S_{1}, S_{2}, \cdots$ be a sequence of finite nonempty sets. For each $i \geqq 2$, let $f_{i}$ be a function from $S_{i}$ into $S_{i-1}$. Then there exists a sequence $s_{1}, s_{2}, \cdots$ such that $s_{i} \in S_{i}$ and $f_{i}\left(s_{i}\right)=s_{i-1}$ for all $i \geqq 2$.

Proof. For convenience, let $S_{0}$ be a set consisting of a single element $s_{0}$ and let $f_{1}$ be the function from $S_{1}$ into $S_{0}$. Let $f_{i j}=f_{i} \circ f_{i+1}$ $\circ \cdots \circ f_{j}$ for $i<j$. Suppose that a sequence $s_{0}, s_{1}, \cdots, s_{n}$ has been found such that for $i \leqq i \leqq n$ and $i<j, f_{i}\left(s_{i}\right)=s_{i-1}$ and $s_{i} \in f_{i j}\left(S_{j}\right)$. (If $n=0, s_{0}$ by itself is such a sequence). To prove the lemma, it suffices by induction to show that such a sequence can be augmented - that is, that there exists $s_{n+1} \in S_{n+1}$ such that $f_{n+1}\left(s_{n+1}\right)=s_{n}$ and $s_{n+1} \in f_{n+1, j}\left(S_{j}\right)$ for $n+1<j$. For this, choose for each $j>n+1$ an element $x_{j}$ of $S_{j}$ such that $f_{n j}\left(x_{j}\right)=s_{n}$; and for $j>n+1$, let $y_{j}=f_{n+1, j}\left(x_{j}\right)$. Then since $S_{n+1}$ is finite, there exists $y$ in $S_{n+1}$ such that $y=y_{j}$ for infinitely many $j$. Now $y=s_{n+1}$ has the desired properties.

THEOREM 2. G has a faithful completely reducible representation of degree $n$ over $K$ if and only if

(1) $G$ is countable; and

(2) each finite subset of $G$ is contained in a finite subgroup $H$ which has a faithful completely reducible representation of degree $n$ over $K .^{3}$

Proof. Suppose first that $G$ has a faithful completely reducible representation $\rho$ in $V$ over $K$. Then $G$ is countable by Theorem 1 . Let $S$ be a finite subset of $G$. Let $V=\sum_{1}^{m} \oplus V_{k}$ where the $V_{k}$ are irreducible $G$-subspaces of $V$. For each $k, G$ has a finite subgroup $H_{k}$ which

2 This lemma is a special case of a theorem of König [3, Theorem 6, p. 81].

3 Malcev, using quite different techniques, shows in [4] that a group $G$, every finitely generated subgroup of which has a faithful representation of degree $n$, has a faithful representation of degree $n$, though possibly over a much bigger field. In this general setting, one has no control over the ground field (as is illustrated by unipotent groups over large fields of nonzero characteristic). 
is irreducible in $V_{k}$. (An argument for this is given in the proof of Theorem 1.) Let $H$ be the subgroup generated by the set $S \cup H_{1} \cup \ldots$ $\cup H_{m} . H$ is finite and contains $S$; and $(\rho \mid H, V)$ is a faithful completely reducible representation of $H$ of degree $n$ over $K$.

Now suppose that $G$ satisfies the conditions (1) and (2). Let $V$ be a vector space over $K$ of dimension $n$. Then we can choose a chain $H_{1} \subseteq H_{2} \subseteq \cdots$ of finite subgroups $H_{i}$ of $G$ such that $G=\cup H_{i}$ and such that for each $i$, the set $S_{i}$ of equivalence classes of faithful completely reducible representations of $H_{i}$ in $V$ over $K$ is nonempty. The $S_{i}$ are finite, and we proceed to define mappings $f_{i}: S_{i} \rightarrow S_{i-1}$ for $i \geqq 2$. For $i \geqq 2$, let $\rho$ be a representative of an element of $S_{i}$. Pass from the representation $\left(\rho \mid H_{i-1}, V\right)$ to the direct sum $\left(\rho^{\prime}, V^{\prime}\right)$ of its composition factors as representations of $H_{i-1}$ over $K$. Then $\left(\rho^{\prime}, V^{\prime}\right)$ is a completely reducible representation of $H_{i-1}$ of degree $n$ over $K$, and we claim that it is faithful. Thus let $I$ be the kernel in $H_{i-1}$ of $\left(\rho^{\prime}, V^{\prime}\right)$. $\rho(I)$ is then a normal unipotent subgroup of $\rho\left(H_{i-1}\right)$. Since $\rho$ is faithful, it follows that $I$ is a $p$-group if $K$ has characteristic $p>0$ and that $I=\{1\}$ if $K$ has characteristic $0 . H_{i-1}$ has a faithful completely reducible representation $\rho_{i-1}$ (since $S_{i-1}$ is nonempty), and the preceding observation shows that $\rho_{i-1}(I)$ is a normal unipotent subgroup of $\rho_{i-1}\left(H_{i-1}\right)$. As a normal subgroup of the completely reducible linear group $\rho_{i-1}\left(H_{i-1}\right), \rho_{i-1}(I)$ is completely reducible $[1$, p. 343]. And as a completely reducible linear unipotent group, $\rho_{i-1}(I)=\{1\}[2$, pp. 775-776]. Since $\rho_{i-1}$ is faithful, $I=\{1\}$. Thus $\left(\rho^{\prime}, V^{\prime}\right)$ is faithful. Therefore $\left(\rho^{\prime}, V^{\prime}\right)$ is equivalent to a representative of a unique element of $S_{i-1}$. Thus the mapping $(\rho, V) \rightarrow\left(\rho^{\prime}, V^{\prime}\right)$ induces a mapping $f_{i}: S_{i} \rightarrow S_{i-1}$. By the lemma, there exists a sequence $s_{1}, s_{2}, \cdots$ such that $s_{i} \in S_{i}$ and $f\left(s_{i}\right)=s_{i-1}$ for $i \geqq 2$. For each $i$, choose a representative $\rho_{i}$ of $s_{i}$; and let $V_{i}$ be the $H_{i}$-module over $K$ defined by the representation $\left(\rho_{i}, V\right)$. (The underlying vector space of $V_{i}$ is $V$; and the action of $H_{i}$ on $V_{i}$ is given by $\rho_{i}$.) Then the construction of the $f_{i}$ and the equations $f_{i}\left(s_{i}\right)=s_{i-1}$ show that for $i \geqq 2, V_{i-1}$ and the direct sum $V_{i}^{\prime}$ of the composition factors of the restriction of $V_{i}$ to $H_{i-1}$ are equivalent as $H_{i-1}$-modules over $K$. For each $i$, choose a decomposition $V_{i}=\sum_{k=1}^{n_{i}} \oplus V_{i, k}$ of $V_{i}$ where the $V_{i, k}$ are irreducible $H_{i}$-submodules of $V_{i}$ over $K$. Then $n_{1} \geqq n_{2} \geqq \cdots$. (If $i \geqq 2$, then $n_{i} \leqq n_{i-1}$; for by the equivalence of $V_{i-1}$ and $V_{i}^{\prime}, n_{i-1}$ is the number of composition factors of the restriction $V_{i} \mid H_{i-1}$ of $V_{i}$ to $H_{i-1}$.) For some $j$, we have $n_{1} \geqq n_{2}$ $\geqq \cdots \geqq n_{j}=n_{j+1}=n_{j+2}=\cdots$. Thus for $i \geqq j+1, n_{i-1}=n_{i}$ and the series

$$
V_{i, 1} \subset V_{i, 1} \oplus V_{i, 2} \subset \cdots \subset \sum_{k=1}^{n_{i}} \oplus V_{i, k}=V_{i}
$$


of $H_{i}$-submodules of $V_{i}$ is in fact a composition series for the restriction of $V_{i}$ to $H_{i-1}$. (For if the above series were to admit an $H_{i-1}$-stable refinement, a composition series for the restriction of $V_{i}$ to $H_{i-1}$ would determine more than $n_{i}$ composition factors, contradicting $n_{i-1}=n_{i}$.) It follows that for $i \geqq j+1$, the representation $V_{i}^{\prime}$ of $H_{i-1}$ over $K$ defined earlier is equivalent to the restriction $V_{i} \mid H_{i-1}$ of $V_{i}$ to $H_{i-1}$. Thus $V_{i-1}$ and $V_{i} \mid H_{i-1}$ are equivalent for $i \geqq j+1$. By suitably modifying the $V_{i}$ successively up to equivalence, we may assume that $V_{i-1}=V_{i} \mid H_{i-1}$ for $i \geqq j+1$. The condition $V_{i-1}=V_{i} \mid H_{i-1}$ for $i \geqq j+1$ is that the underlying vector spaces of the $V_{i}$ coincide for $i \geqq j+1$; and that if $\rho_{i}: H_{i} \rightarrow \operatorname{Hom}_{K}\left(V_{i}, V_{i}\right)$ denotes the action of $H_{i}$ on the underlying vector space $V_{i}$ of $V_{i}$ for all $i$, then $\rho_{s} \mid H_{r}=\rho_{r}$ for $j+1$ $\leqq r \leqq s$. Now if $\rho=U_{r \geq j+1} \rho_{r}$ (that is, if $\rho$ is the function with domain $G=\bigcup_{r \geqq j+1} H_{r}$ defined by $\rho(g)=\rho_{r}(g)$ if $g \in H_{r}$ and $\left.r \geqq j+1\right)$, then $\rho$ is a faithful completely reducible representation of $G$ of degree $n$.

\section{BIBLIOGRAPHY}

1. C. W. Curtis and I. Reiner, Representation theory of finite groups and associative algebras, Interscience, New York, 1962.

2. E. Kolchin, On certain concepts in the theory of algebraic matric groups, Ann. of Math. (2) 49 (1948), 774-789.

3. D. König, Theorie der Endlichen und Unendlichen Graphen, Chelsea, New York, 1950.

4. A. I. Malcev, On the faithful representations of infinite groups by matrices, Mat. Sb. 8 (1940), 405-422; English transl., Trans. Amer. Math. Soc. (2) 45 (1965), 1-18.

YALE UNIVERSITY 